\title{
EFFETTI SISTEMICI \\ E POLITICA INTERNAZIONALE
}

\author{
di Filippo Andreatta
}

Glenn Snyder, Alliance Politics, Ithaca, N.Y., Cornell University Press, 1997, pp. 414, Isbn: 0-8014-3402-5.

Robert Jervis, System Effects, Princeton, NY, Princeton University Press, 1997, pp. 309, Isbn: 0-691-02624-6.

Questi due recenti lavori di importanti studiosi statunitensi dimostrano ancora una volta la vitalità e la creatività della disciplina delle relazioni internazionali. Si tratta infatti di opere di grande respiro e rigore teorico che introducono nel dibattito accademico ipotesi ed analisi alquanto avvincenti. Il libro di Snyder si concentra su uno degli aspetti essenziali della politica internazionale - le alleanze - e riesce nell'intento di affrontare approfonditamente un argomento che, paradossalmente, è invece rimasto piuttosto trascurato nella letteratura specializzata. A parte la letteratura politologica sulle coalizioni ed i beni pubblici, e la letteratura più classica sulle relazioni internazionali, non ci sono infatti analisi recenti in materia. Il volume di Jervis è invece meno ambizioso in quanto non si presenta come un'analisi compiuta su uno degli aspetti principali della disciplina. Ciò nonostante, l'autore cerca di proporre una chiave di lettura sistemica di tutta la politica internazionale risultando così non meno rilevante del libro di Snyder. La ricchezza del background culturale di Jervis e la sua capacità di sintesi - che già si erano espresse in campi diversi quali la teoria della deterrenza nucleare, l'importanza delle variabili tecnologiche, l'analisi psicologica delle decisioni e le grandi metafore strategiche della diplomazia contemporanea - assumono in questo caso una forma matura che permette all'autore di attingere proficuamente da altre discipline quali la biologia e l'ingegneria am- 
bientale senza mai perdere di vista l'intento di spiegare fenomeni sociali complessi.

I due volumi trattati offrono una risposta, convincente anche se ancora interlocutoria, ad una delle maggiori questioni che hanno caratterizzato il dibattito teorico nelle relazioni internazionali negli ultimi vent'anni. Dalla pubblicazione di Theory of International Politics di Waltz nel 1979, la grande maggioranza dei contributi si è concentrata a sostenere la tradizione del «realismo strutturale» o a criticarne l'eccessiva parsimonia nel numero di ipotesi, senza peraltro riuscire a trovare una valida alternativa. Nei termini astratti dei principi di Kuhn e Lakatos, sono state trovate numerose eccezioni al paradigma waltziano senza che queste fossero spiegate in modo soddisfacente da un altro paradigma di uguale eleganza e coerenza, impedendo dunque l'insorgenza di un paradigm shift. In particolare, è stata evidenziata quella che si può definire come «l'insufficienza della struttura», in altre parole l'impossibilità di spiegare risultati variabili come quelli che caratterizzano la politica internazionale con caratteristiche esclusivamente strutturali - e quindi costanti nel tempo o omogenee nello spazio - del sistema internazionale. Come ha sinteticamente espresso un sottile studioso inglese: «Se l'anarchia portasse inevitabilmente alla guerra, allora gli Stati europei non avrebbero conosciuto la pace almeno dalla fine del medioevo» (Hinsley 1968, 324). L'anarchia cioè può essere una causa permissiva, ma non sufficiente, per il conflitto. Nonostante le sue ambizioni deterministiche, la teoria neorealista è al contrario vulnerabile proprio perché eccessivamente indeterminata.

Anche se ha avuto il notevole merito di sottolineare l'importanza della struttura anarchica del sistema internazionale $\mathrm{e}$ l'inopportunità di prescindere da questa rendendola una specie di «stele di Rosetta» delle relazioni internazionali, l'analisi waltziana non ha saputo in effetti trovare una risposta definitiva ad annosi dibattiti su quali siano le specifiche condizioni che effettivamente favoriscano la pace o la guerra $o$, più in generale, la cooperazione o il conflitto. Ad esempio, le vivaci diatribe accademiche tra sostenitori della stabilità dei sistemi bipolari piuttosto che di quelli multipolari testimoniano l'indeterminatezza di una teoria esclusivamente strutturale (Andreatta 1997). Waltz stesso, nella sua classica perorazione del bipolarismo come forma più stabile di distribuzione di potenza, accusa i sistemi multipolari di una duplice sindrome - il buckpassing, in altre parole 
la tendenza delle alleanze multipolari a disgregarsi a causa del free riding proprio della logica dell'azione collettiva; e il chainganging, ovvero la tendenza ad irrigidirsi eccessivamente delle stesse alleanze per paura di incentivare la defezione delle proprie controparti - senza peraltrø suggerire quali fattori favoriscano l'emergere di una piuttosto che dell'altra. Lavori successivi, tra cui anche alcuni interventi di Waltz stesso, hanno recuperato come un addizionale fattore causale la tecnologia, che però come caratteristica non strutturale (in quanto attributo delle unità ed in quanto variabile per eccellenza) ha il vizio di contraddire - indebolendolo - lo stesso impianto strutturale della teoria.

In termini propositivi, due sono state le strade più seguite rispetto al problema dell'insufficienza della struttura. Da un lato, si sono recuperate delle variabili esogene (altrimenti chiamate riduzioniste) che contribuirebbero a spiegare $\mathrm{i}$ risultati della politica internazionale in base alle caratteristiche delle unità: la propensione della loro politica estera (revisionista o status quo) o la loro struttura interna (democratica o meno). Stati rivoluzionari, espansionisti e dotati di una legittimità diversa dagli altri porterebbero quindi ad un sistema più instabile di Stati più moderati ed omogenei tra loro. Il vantaggio di queste teorie più articolate consiste in una maggiore capacità descrittiva di una realtà complessa e variegata. Il maggiore problema è che la descrizione non costituisce di per sé una spiegazione, dal momento che rimane comunque da spiegare come e perché le varie unità si comportino in modo diverso. Le teorie riduzioniste non affrontano inoltre il problema di quei risultati «paradossali» che rappresentano il nocciolo più interessante dell'intera disciplina delle relazioni internazionali, ovvero quei casi in cui anche unità con intenzioni moderate finiscono col comportarsi in modo disfunzionale alla stabilità del sistema o in cui unità dichiaratamente «eterogenee» si comportano invece in modo omogeneo alle altre.

Dall'altro lato, si è seguita la strada post-moderna del costruttivismo, in cui si è attribuito un valore causale a variabili ideologiche e normative. Sistemi internazionali in cui la guerra è ammessa come uno strumento legittimo della politica estera tenderebbero, ad esempio, a produrre un maggior numero di conflitti armati di sistemi in cui la forza è considerata come un mezzo inaccettabile per fini politici. Questa strada, in voga soprattutto tra gli studiosi della cosiddetta «teoria critica», pre- 
senta però due gravi problemi di carattere metodologico. In primo luogo, l'argomentazione è di tipo circolare, dal momento che non è chiaro se la pace sia il prodotto del pacifismo o viceversa. In secondo luogo, al contrario che nella ricerca storica che è tesa ad identificare i caratteri particolari di un evento, è pericoloso utilizzare variabili così sfuggenti come quelle culturali ed intellettuali per processi politici che richiedono uno sforzo di generalizzazione. Si corre altrimenti il rischio di affermare che «i lillipuziani si comportano da lillipuziani perché sono lillipuziani», invece di trovare delle ipotesi effettivamente confrontabili con un'ampia gamma di circostanze empiriche.

La strada percorsa dagli autori dei due saggi qui in discussione è sì quella di trovare delle nuove variabili esplicative, ma di organizzarle in modo più chiaro ed in una cornice utile allo sviluppo dei modelli teorici. Rispetto alla semplice definizione di Waltz di struttura (che comprendeva i tre elementi dell'anarchia, dell'uguaglianza funzionale degli Stati e della sola distribuzione di potenza come variabile), questi lavori introducono una maggiore ricchezza analitica con il minimo costo in termini di parsimonia. Non vi è infatti l'invocazione di un deus ex machina o di variabili esogene al sistema internazionale per spiegare la varianza di risultati al suo interno; né si rinuncia alla visione tradizionale di un sistema internazionale che filtra, media e distorce, come un prisma, le intenzioni degli Stati. Anche le migliori intenzioni possono infatti portare a risultati perversi se, ad esempio, la tolleranza e la ricerca del compromesso incentivano gli sforzi espansionistici di un aggressore. Al contrario, cattive intenzioni non portano necessariamente alla guerra se la politica aggressiva di uno Stato scatena delle controreazioni che la bilanciano. Il maggiore contributo di questi lavori è il tentativo di guardare dentro questa «scatola nera» per capire più precisamente cosa avvenga al suo interno.

In particolare, entrambi ammettono che la struttura del sistema non è determinante da sola e suggeriscono quindi un nuovo tipo di processi (le relazioni per Snyder e i feedback per Jervis) che aiutino a spiegare le variazioni nei risultati (vedi fig. 1). Questi processi non sono però delle altre variabili indipendenti che attenuerebbero la parsimonia della teoria, ma piuttosto delle variabili intervenienti gerarchicamente, sottoposte alla struttura internazionale e dotate ciò nonostante di una propria autonoma capacità di influenzare i risultati. In altre parole, non si mira in questi lavori a sostituire il valore causale della struttu- 


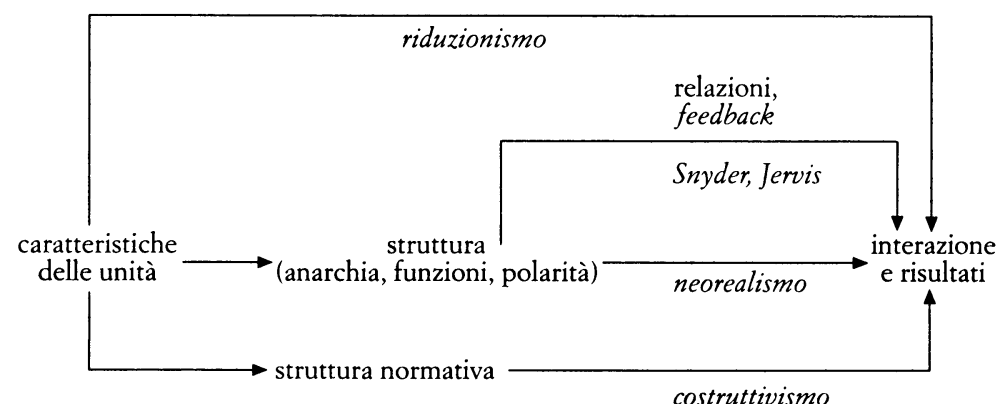

FIG. 1. Relazioni causali in varie tradizioni di ricerca.

ra anarchica del sistema internazionale, ma si vuole anzi sottolineare come l'anarchia possa, a seconda di alcune situazioni diverse (le variabili intervenienti appunto), causare sia risultati cooperativi che di conflitto. I due autori si concentrano quindi su una prima analisi di quali siano le condizioni a favore dell'uno o dell'altro tipo di risultato.

Le relazioni - bilaterali e multilaterali - sono quindi variabili intervenienti le cui caratteristiche sono influenzate dalla struttura del sistema internazionale, ma che hanno un proprio valore causale nei confronti dei risultati effettivi. In altre parole, le relazioni sono elementi del sistema internazionale attualizzati in un preciso ambito storico e geografico, ovvero il mezzo attraverso cui la struttura influenza le decisioni degli Stati e le interazioni tra loro. Le relazioni sono quindi elementi sistemici ma non strutturali, in quanto possono cambiare nel tempo e nello spazio ed in quanto non influenzano contemporaneamente tutti gli Stati ma solo un sottogruppo di questi. Allo stesso tempo, le relazioni non sono nemmeno degli attributi delle unità perché non fanno parte delle loro intrinseche caratteristiche, ma anzi vengono in qualche modo subite da queste come dei vincoli alla loro libertà d'azione. Mentre dunque la struttura anarchica sarebbe il generico contesto della politica estera degli Stati, le relazioni sarebbero il contesto specifico di ciascuna singola decisione.

Per chiarire il percorso dei vari nessi causali e l'utilità dell'idea di relazione, si prenda in esame il concetto di potere sia per la sua importanza nelle relazioni internazionali sia per la sua nota scarsa predisposizione ad una definizione precisa. Il con- 
cetto di potere può quindi venire scomposto in vari elementi. In primo luogo, a livello degli attributi delle unità, vi sono le risorse degli Stati, la somma dei mezzi tangibili ed intangibili a loro disposizione che ne determina la posizione rispetto agli altri. In secondo luogo, la struttura determina la natura del potere degli Stati, che in una situazione di assenza di gerarchia è sovrana, cioè indipendente e priva di differenziazione funzionale. Tutti gli Stati infatti sono dotati di un potere qualitativamente uguale sul territorio di loro competenza anche se in diverse quantità. In terzo luogo, queste risorse sovrane si trasformano in capacità, ovvero in una relazione (appunto) di potenza tra specifici Stati. Le risorse degli Stati non hanno infatti un valore astratto ed assoluto e devono venire comparate con quelle delle loro controparti per assumere un valore concreto. La risorsa di un esercito di 10.000 soldati può trasformarsi in una capacità elevatissima se gli altri Stati sono dotati di forze armate di 1.000 uomini o in una capacità alquanto scarsa se il livello degli altri eserciti è di 100.000. In quarto ed ultimo luogo, il potere viene effettivamente esercitato in un'interazione in cui una delle controparti ottiene che l'altra si comporti in modo diverso (e più compatibile con i propri obiettivi) da quello in cui si sarebbe comportata altrimenti. In altre parole, la relazione si limita ad una capacità potenziale che influenza e vincola attivamente le interazioni tra gli attori, ma non è necessariamente concretizzata e deve pertanto essere distinta dall'effettivo esercizio del potere.

Snyder menziona quattro tipi di relazione che filtrano gli effetti della struttura sulle effettive interazioni tra gli Stati. Gli allineamenti, che più da vicino riguardano l'analisi dell'autore sulle alleanze, sono le aspettative sull'amicizia e inimicizia tra i vari Stati che determina l'atteggiamento di base - benevolo o competitivo - nei rapporti bilaterali. Queste aspettative sono relazioni in quanto non sono elementi della struttura in senso stretto ma non sono neanche esclusivamente dipendenti dal concreto comportamento diplomatico degli Stati. E infatti possibile immaginare una formale alleanza che non viene ritenuta credibile e al contrario un raggruppamento di Stati che probabilmente combatterà insieme in caso di guerra anche senza un esplicito trattato. Un'altra importante relazione è la configurazione degli interessi degli Stati, sia quelli di tipo strategico inerenti alla posizione dello Stato rispetto agli altri, sia quelli di tipo intrinseco legati agli specifici obiettivi geopolitici, economi- 
ci ed ideologici dello Stato in questione. Il terzo tipo di relazione è quello delle capacità, ovvero il grado in cui le risorse di uno Stato si possono tradurre in potenzialità per modificare il comportamento delle controparti. $\grave{E}$ in questa categoria che è possibile concepire correttamente il ruolo della tecnologia come variabile sistemica ma non strutturale capace di alterare gli incentivi e i vincoli degli Stati. L'ultima relazione cui si riferisce Snyder è quella dell'interdipendenza, cioè il grado di necessità che uno Stato ha degli altri in generale per raggiungere $i$ propri obiettivi, nonché del livello al quale specifici Stati soddisfano questo bisogno.

Anche se Snyder non li menziona, è possibile immaginare altri tipi di relazione. Per esempio, la cornice istituzionale in cui operano gli Stati delimita i comportamenti considerati accettabili da quelli inaccettabili e pertanto influenza anch'essa le valutazioni degli Stati. Come le altre relazioni, la cornice istituzionale non è strutturale in quanto è variabile nel tempo e nello spazio; è tuttavia un elemento sistemico dal punto di vista degli Stati che ne subiscono i vincoli senza poterli direttamente alterare. Jervis sottolinea l'importanza di questi elementi istituzionali quando, richiamando il fondamentale lavoro storico di Schroeder sulla politica internazionale attorno al Congresso di Vienna (Schroeder 1994), afferma che forse «il perseguimento del proprio autointeresse può portare stabilità e un certo livello di pace solo se è vincolato da una concezione normativa che limiti il comportamento predatorio» (p. 137).

L'assenza di questi vincoli contribuirebbe quindi ad un effetto negativo dell'anarchia sulla stabilità, mentre la loro presenza filtrerebbe gli stessi effetti con un esito diverso e più positivo. Nel loro insieme quindi, le relazioni definiscono gli obiettivi e i mezzi possibili per gli Stati nei confronti degli altri e influenzano risultati di un tipo piuttosto che di un altro. Nonostante tutte le relazioni siano influenzate dall'anarchia perché i concetti di capacità, di interdipendenza o di vincolo istituzionale avrebbero un carattere ben diverso in un regime gerarchico, risultati cooperativi o conflittuali dipendono quindi dalla natura delle relazioni in atto in un preciso contesto storico e geografico.

Anche Jervis ci ricorda l'importanza analitica degli effetti sistemici, da cui non si può prescindere per comprendere gli eventi internazionali. Un sistema è tale se: $i$ ) vi è un certo numero di elementi interconnessi per cui un cambiamento in uno 
di questi elementi produce cambiamenti in altre parti del sistema; ii) il sistema nel suo complesso esibisce proprietà diverse da quelle delle unità. È su questa diversità che Jervis si concentra per spiegare i risultati talvolta paradossali e quasi sempre non intenzionali che caratterizzano le relazioni internazionali. La complessità delle interconnessioni in un sistema frustra infatti comportamenti eccessivamente volontaristici in quanto è quasi impossibile agire su un singolo elemento di un sistema senza alterare gli altri, scatenando una catena di azioni e di reazioni che è difficile controllare o anche solo prevedere. Questo approccio ricorda la critica delle economie pianificate di Hayek, che ammoniva come politiche di questo tipo «anche se mirano a conseguire un particolare e prevedibile risultato, potrebbero inibire altri risultati. Non saremo mai completamente consci di tutti i costi che il perseguimento di un risultato particolare comporta quando è perseguito tramite un'interferenza» (Hayek 1982, 75).

Ci sono quindi tre tipi di interazioni che bisogna prendere in considerazione. In primo luogo, le interazioni tra un'azione e le condizioni del sistema in cui l'azione ha luogo. Il medesimo comportamento, ad esempio l'intervento militare verso un vicino più debole, può avere effetti molto diversi se ci si trova in una rigida ed esasperata competizione bipolare o in un più permissivo contesto multipolare. Gli Stati Uniti, ad esempio, sono intervenuti a Cuba diverse volte prima della guerra fredda, ma hanno evitato comportamenti drastici durante la crisi dei primi anni '60. In secondo luogo, le strategie degli attori sono interconnesse tra loro per cui una determinata azione può portare ad una reazione inaspettata. Jervis cita un aneddoto della sua gioventù, in cui un suo compagno di corso aveva comprato un lucchetto per proteggere il suo armadietto finendo però con l'attirare l'attenzione dei ladri che pensavano che l'unico armadietto contenente oggetti di valore fosse proprio quello più protetto. In tutte le scienze sociali è nota la sindrome dell'azione collettiva secondo la quale un'intensificazione dei propri sforzi di produzione di un bene pubblico, tesa ad incoraggiare gli altri a fornire il loro contributo, potrebbe invece avere un risultato perverso incentivando gli altri al free-riding o al moral bazard ${ }^{1}$.

1 L'azzardo morale è quel meccanismo per cui una misura tesa a contenere gli effetti negativi di un comportamento disfunzionale può in realtà contribuire a generarlo perché ne riduce gli eventuali costi. 
Questo è il tipo di interazione cui si riferiva Clausewitz quando spiegava come un'azione militare soggetta a rigidi obiettivi politici potesse degenerare, a causa dello scontro con la volontà del nemico e dei suoi imprevedibili effetti, in una guerra senza limiti. In terzo luogo, le azioni degli attori possono esse stesse cambiare le condizioni sistemiche in cui hanno luogo. Ad esempio, un'azione drammatica come l'invasione del Kuwait può modificare radicalmente il livello di tensione internazionale. Anche se prima dell'invasione il sistema reduce dalla guerra fredda sembrava essere tendenzialmente moderato e permissivo, e quindi poteva far pensare ad una reazione internazionale limitata, l'Iraq aveva sottovalutato gli effetti del proprio gesto, che ha invece modificato in un batter d'occhio la natura del sistema, creando le condizioni per una reazione massiccia.

Questi tre tipi di interazioni - tra sistema ed unità, tra unità e unità e tra unità e sistema - rendono molti effetti sistemici non lineari. Mentre un effetto lineare mantiene una relazione proporzionale tra input e output, un effetto non lineare ha una reazione sproporzionata al suo input almeno in certe circostanze. Come sanno tutti i figli, la violazione delle regole di comportamento impartite dalla madre è tollerata fino ad un certo punto, oltre il quale scatta una punizione che spesso non è commisurata all'ultima violazione che ha scatenato la reazione. Nella politica internazionale, uno sforzo moderato di incrementare la propria capacità di difesa tramite il riarmo può effettivamente risultare in una condizione di maggior sicurezza, mentre un riarmo eccessivo può generare controreazioni da parte degli altri Stati che potrebbero peggiorare le condizioni iniziali dello Stato in questione, secondo la logica del dilemma della sicurezza. Uno dei principali conduttori di non linearità in un sistema è il feedback, un processo interveniente in cui «la causazione è reciproca o circolare piuttosto che unidirezionale». Un feedback è infatti presente quando «un cambiamento in un elemento o in una relazione [tra elementi] altera altri [elementi o relazioni], che a loro volta influenzano quello originale» (p. 125).

Gli effetti di retroazione possono essere di due tipi. Sono positivi quando amplificano l'input - come quando il panico si scatena tra la folla generando un proprio momentum - e sono negativi quando invece ne riducono gli effetti. In politica internazionale, il feedback negativo per eccellenza è costituito dalla Balance of Power, secondo cui tanto più uno Stato sarà potente e minaccioso per gli altri, tanto più questi mobiliteranno risorse 
e si coalizzeranno contro il primo. Questo meccanismo è un effetto sistemico in quanto non dipende né dalle caratteristiche né dalle intenzioni delle unità: «Gli Stati non perseguono l'equilibrio; i vincoli non sono interni nel senso che ciascuno Stato crede che dev'essere vincolato. La moderazione e la stabilità emergono piuttosto perché l'ambizione [dell'uno] controlla l'ambizione [dell'altro] e l'interesse bilancia l'interesse» ( $\mathrm{p}$. 132). L'equilibrio di potenza deriva quindi dalla condizione anarchica e dalla volontà di sopravvivenza delle unità, che sono costrette al self-belp dalla mancanza di un'autorità capace di proteggerli e che tendono ad allearsi contro il più forte perché rappresenta la minaccia più impellente, e sarà pertanto la caratteristica dominante della politica internazionale e la fonte primaria della sua stabilità ${ }^{2}$.

Ciò nonostante, la politica internazionale non è immune da feedback positivi che ne possono minare la stabilità. Questo si verifica tramite meccanismi di domino, ovvero quando gli Stati riescono ad accumulare un vantaggio iniziale e ad amplificarlo fino a quando diventa determinante, anche perché altri Stati seguono il bandwagon, salendo sul carro del vincitore. Questi processi, e persino la sola percezione che siano in atto, anche se errata, possono generare spirali di instabilità per cui tutti gli Stati cercano di accaparrarsi ogni piccolo vantaggio. Questi episodi sono però relativamente rari e non contraddicono la generale tendenza all'equilibrio, anche se sottolineano la complessità e l'incertezza delle relazioni internazionali. Non è infatti escluso che relazioni non lineari possano portare sia ad un feedback positivo che ad uno negativo. Ad esempio, gli iniziali successi di una potenza emergente potrebbero attirare nuove risorse e nuovi alleati anche se, oltre un certo punto, potrebbero scatenare preoccupazioni tali da creare le condizioni per un bilanciamento.

Le non linearità e le complessità del sistema internazionale si ripercuotono sui comportamenti degli Stati e sulle relazioni tra loro. In particolare, Jervis sottolinea come spesso gli allineamenti seguano una logica triangolare piuttosto che bilaterale. In

2 Jervis, al contrario della maggior parte della letteratura, intende la stabilità né come pace né come persistenza strutturale, ma più correttamente come la tendenza di un sistema all'omeostasi, ovvero a ritornare in equilibrio dopo che uno shock esogeno ha posto il sistema in disequilibrio. È infatti possibile che il ritorno alla stabilità di un sistema richieda proprio una guerra, mentre è possibile immaginare un sistema stabile ma di scarsa durata, a causa di ragioni esogene straordinarie. 
altre parole, gli Stati non sceglierebbero i propri partner in base alle comuni ed intrinseche affinità di interesse, ma a causa di una comune inimicizia con una terza parte. «Il fattore più forte [a favore della stabilità] è un comune nemico, che può far superare anche le più forti divergenze tra gli attori» (p. 221). È in questo modo che si possono spiegare, ad esempio, i singolari allineamenti della seconda guerra mondiale tra democrazie occidentali e comunismo totalitario, che non avrebbero senso fuori dal contesto sistemico in cui hanno avuto luogo.

Non solo le alleanze devono essere viste attraverso il prisma del sistema, ma bisogna anche considerare i loro effetti di retroazione sul sistema stesso. Le alleanze infatti sono generate da comuni interessi, ma sono anche in grado di generare interessi autonomamente, secondo la logica della causalità mista. Le alleanze infatti non definiscono solo la nascita di un'amicizia, ma possono anche comportare nuove inimicizie, ad esempio contro gli alleati dello Stato contro cui è rivolta l'alleanza, e nuove amicizie, ad esempio con gli alleati dell'alleato. Processi di polarizzazione possono quindi estremizzare ed irrigidire allineamenti iniziali molto più sfumati e indeterminati. Per illustrare questo processo, Jervis utilizza l'esempio delle origini della prima guerra mondiale, che è tra l'altro anche la fonte dell'evidenza empirica del libro di Snyder. Senza gli effetti sistemici, sarebbe infatti difficile comprendere la configurazione degli allineamenti di quel periodo, soprattutto nel caso della Gran Bretagna che Jervis suggerisce fosse «dalla parte sbagliata» (p. 243).

Gli interessi intrinseci della Gran Bretagna non si scontravano infatti con quelli della Germania, che non era dotata di un impero, ma semmai proprio con quelli di Francia e Russia, come dimostrano le ripetute crisi in Africa e in Asia della fine del secolo. Berlino infatti non si aspettava che la Gran Bretagna combattesse realmente e ha sperato in un'alleanza con Londra o almeno nella sua neutralità fino alla fine. In ogni caso, la grande potenza con cui gli interessi inglesi erano meno in conflitto era l'Austria-Ungheria, che invece combatté sul lato opposto. La necessità britannica di diminuire il numero dei propri nemici potenziali in un'era di declino relativo, che aveva portato all'entente con Parigi, ha poi scatenato le reazioni del nemico per eccellenza della Francia - la Germania - che a loro volta hanno provocato per controrezione l'entente con l'alleato della Francia, la Russia, ingenerando in Germania la nota sindrome d'accerchiamento che ha poi irrigidito la sua diplomazia. 
Snyder articola ancor più sofisticatamente queste considerazioni sistemiche. Anch'egli sottolinea l'importanza delle triangolazioni con terze parti nei rapporti tra alleati, che sono quindi solo in parte governati dall'intrinseca comunanza di interessi, tanto che «le alleanze non possono essere studiate separatamente dalle inimicizie che le catalizzano» (p. 50). Nel suo modello a $\mathrm{N}$-giocatori che ricostruisce $\mathrm{i}$ meccanismi di formazione delle alleanze, Snyder basa la sua analisi su una semplice comparazione tra costi e benefici illustrando come i meccanismi di bilanciamento siano preminenti. In parole povere, il forte tende a non allearsi col forte contro il debole, ma col debole contro il forte o al limite col debole contro un altro debole (p. 60). La questione è però complicata dal fatto che, a meno di non avere una straordinaria distribuzione dei pay-off, la specifica alleanza che effettivamente si forma in un sistema multipolare è indeterminata mentre, in piena sintonia con l'argomentazione di Jervis, anche quelle alleanze che non si formano influenzano quelle che si formano effettivamente in quanto ciascun partner valuta anche tutte le alternative a propria disposizione prima di decidere. La disponibilità di alleanze alternative, anche qualora non venga concretizzata, è quindi il principale vantaggio che può venire utilizzato con i propri partner per ottenere un accordo più favorevole. Ne consegue inoltre che la mancanza di valide alternative diplomatiche può costituire una significativa debolezza.

L'insufficienza della struttura è anche dimostrata proprio dalla presenza delle alleanze stesse. Se gli allineamenti fossero interamente determinati dalla struttura, infatti, le alleanze sarebbero inutili (nel caso in cui gli interessi fossero già convergenti le aspettative di supporto non avrebbero bisogno di conferme), o sarebbero vuote promesse se gli interessi fossero invece divergenti. È proprio perché le varie relazioni complicano la struttura e la rendono meno lineare e prevedibile che le alleanze sono, al contrario, utili e preziose. L'indeterminatezza dell'anarchia ha infatti bisogno di vincoli aggiuntivi per produrre una configurazione trasparente e stabile. L'utilità delle alleanze è molteplice. In primo luogo, anche quando ratificano un accordo già solido a causa di interessi comuni, le alleanze possono fungere da meccanismo di coordinamento per ottimizzare l'allocazione dei compiti. Senza un'autorità garante degli accordi, nell'arena internazionale la cooperazione non è infatti scontata nemmeno quando le preferenze sono convergenti. In secondo 
luogo, le alleanze producono informazioni precisando l'identità degli amici e dei nemici. Le alleanze precludono ai propri alleati delle alternative incompatibili e sanciscono una scelta tra varie opzioni. Anche qualora gli alleati non avessero la necessità di ribadire formalmente una loro convergenza di interessi, le alleanze potrebbero rivelarsi utili per segnalare a terze parti, e principalmente all'avversario contro cui sono rivolte, la reciproca volontà di supporto, generando un deterrente. In terzo luogo, le alleanze possono aumentare (anche se non garantire) la probabilità che il supporto effettivamente si materializzi, visto che a causa di considerazioni di reciprocità e di reputazione gli Stati preferiscono non rinnegare la parola data in un accordo solenne (pp. 351-363).

La ragione delle alleanze scaturisce quindi dalla necessità di trovare forme autonome di sicurezza e dall'incertezza generata dall'anarchia, che a seconda delle relazioni attraverso cui è filtrata può produrre risultati più o meno stabili. La causa stessa delle alleanze è però anche il suo principale problema. La stessa incertezza anarchica è infatti fonte della possibilità che gli impegni alla fine vengano disattesi e governa quindi la manutenzione delle alleanze e i rapporti di forza all'interno di queste. Il cosiddetto alliance security dilemma, che già Snyder aveva identificato in precedenti lavori, è qui sviluppato per esteso. In sintesi, gli alleati sono tormentati da una duplice prospettiva. Da un lato temono di venire abbandonati dai propri partner nel momento del bisogno. Dall'altro hanno paura di venire intrappolati, ovvero di essere costretti a combattere per onorare i loro impegni anche quando non sono i loro interessi ad essere in gioco, ma quelli dell'alleato. Il dilemma nasce dal fatto che le misure di rassicurazione tese ad evitare l'abbandono aumentano drasticamente i rischi di intrappolamento, e viceversa. Si tratta quindi di un trade-off tra due diversi valori: la propria autonomia e la propria sicurezza. Sono le relazioni prevalenti tra gli alleati che determinano il punto ottimale in questa scelta, ed in particolare il reciproco livello di dipendenza verso i propri alleati, il commitment, ovvero l'interesse a difendere gli alleati anche indipendentemente dagli accordi formali, e gli specifici interessi in gioco in un preciso momento. Tanto più un alleato sarà dipendente $o$ committed, tanto più sarà facile per i suoi partner giocare sulle sue paure di abbandono per intrappolarlo a perseguire i loro interessi. Al contrario, tanto più le preferenze di un alleato saranno intense rispetto agli specifici interessi in gioco, tanto più sarà 
credibile la sua minaccia di abbandonare gli altri se verrà disatteso, e pertanto gli altri tenderanno ad aiutarlo.

Il valore di questi due importanti libri è esaltato dal loro tempismo. Al mondo bipolare della guerra fredda è infatti seguito un mondo multipolare in cui le considerazioni sistemiche e le dinamiche delle alleanze assumono un maggiore rilievo. In un sistema centrato su due grandi blocchi, infatti, le identificazioni delle amicizie e delle inimicizie sono ben più stabili, mentre gli effetti delle triangolazioni sono estremamente ridotti a causa dell'assenza di controparti. Al contrario, in un sistema pluripolare, le amicizie e le inimicizie sono meno assolute e permettono una maggiore libertà di scelta anche a causa della presenza di molteplici allineamenti alternativi e della minore intensità delle minacce. Il lavoro degli autori è quindi molto più rilevante per la politica contemporanea di quanto non lo sarebbe stato prima della caduta del muro di Berlino.

I concetti di relazione, feedback, trade-off e non linearità che formano la spina dorsale dell'analisi di Jervis e Snyder, ricordano la tradizionale cautela che i pensatori classici sulle relazioni internazionali utilizzavano sia nell'analisi sia nella prescrizione. La frustrazione del volontarismo e dell'intenzionalità, la pluralità e l'ambivalenza degli effetti sistemici devono stimolare da parte degli accademici uno sforzo d'umiltà per evitare generalizzazioni eccessive e teorie semplicistiche. La complessità del sistema internazionale richiede invece, da parte di chi pratica la politica internazionale, una consapevolezza che la libertà di scelta non è per nulla eliminata nelle relazioni tra Stati, ma che deve essere utilizzata con responsabilità nei rapporti con gli altri, con prudenza negli strumenti e con moderazione nei fini se si vogliono evitare le peggiori conseguenze dell'anarchia. L'esaltazione degli interessi nazionali o delle varie preferenze ideologiche a prescindere dalle considerazioni di carattere sistemico potrebbe altrimenti rivelarsi disastrosa.

\section{Riferimenti bibliografici}

Andreatta, F. (1997), Configurazione polare e stabilità del sistema internazionale: sistemi bipolari e multipolari a confronto, in «Quaderni di Scienza della Politica», n. 2, pp. 175-200.

Hinsley, F.H. (1968), Power and the Pursuit of Peace, Cambridge, Cambridge University Press. 
Schroeder, P. (1994), The Transformation of European Politics, $1763-$ 1848, Oxford, Oxford University Press.

Von Hayek, F. (1982), Law, Legislation and Liberty, Chicago, University of Chicago Press, trad. it. Legge, legislazione e libertà, Milano, Il Saggiatore, 1986.

Waltz, K.N. (1979), Theory of International Politics, Reading, Mass., Addison-Wesley, trad. it. Teoria della politica internazionale, Bologna, Il Mulino, 1986. 